\title{
Experimental Study on the Detection of Tropical Fruit Quality Based on Gas Identification Technology
}

\author{
Yufei Wang ${ }^{\mathrm{a}}$, Wanzhen Zhang ${ }^{\mathrm{b}}$ and Kun Zhang ${ }^{\mathrm{c}^{*}}$ \\ College of Computer Engineering, Hainan Tropical Ocean University, Sanya, Hainan, 572022, China \\ a1576949369@qq.com, b107689990@qq.com, 'zk0588@163.com \\ ${ }^{*}$ The corresponding author
}

Keywords: Gas identification; Electronic nose; Agriculture products; Tropical fruits; Algorithm analysis

\begin{abstract}
The gas identification technique based on, real-time monitoring of electronic nose detection technology as the main research method, main products for Hainan tropical agricultural products, tropical fruits in storage and transportation of quality change for the purpose, to select the object, by electronic nose technology equipment for experimental test, were analyzed and compared with BP, PCA-BP, LDA-BP neural network algorithm, to obtain the objective data, to analyze the recognition performance and stability of the key technology of the electronic nose system.
\end{abstract}

\section{Introduction}

Quality detection of agricultural products has been subjected to social concern, food safety has become a new hot spot, the Hainan tropical agricultural products mainly in tropical fruit as the main products, tropical fruits in Hainan also has been highly popular and favorite of the people throughout the country, especially in the winter, fruit market is more dominant role, for a long time in storage and transport all kinds of fruit quality change is always important, which determines the market fruit quality and economic benefits. The optimal period of tropical agricultural products regardless of fruits, vegetables and other agricultural products, in the process of storage and transportation will release some of the characteristics of the gas, such as ammonia, ethanol, hydrogen sulfide and other volatile organic gas. Through these gas concentration, density index can analyze product quality changes and storage, transport through on the real-time monitoring and detection to monitor the change of state, to take the necessary measures, to ensure the quality of agricultural products, reduce the loss of value. Simulation of human olfactory perception by electronic nose. In the nineties of the 20th century, Bartlett of the University of Warwick in England Gardner and the University of Southampton first proposed "electronic nose," a word, which is defined as: "a class by a part of the selective chemical sensor array and the pattern recognition system is composed of, through the pattern recognition function of sensor part of the specificity and system for detection of simple or complex smell of electronic equipment". The market mainly to foreign products of the main, and the price is more expensive, mostly around tens of thousands, hundreds of thousands of dollars, due to the needs of the development and application of sensor technology, microelectronics and MEMS technology, electronic nose system products also subsequently to small volume, low cost, practical trend of development, its market prospects very well.

We can through to market existing electronic nose system design and technology improvement to enable it to fully scientific experiments, by choosing is representative of mango, banana, papaya, Sakyamuni and tropical fruits and the of quality recognition experiments, corruption process monitoring experiment through the income obtained experimental data processing, comparison and analysis of the detection performance, valuable conclusions are obtained. 


\section{Hardware Equipment and the Composition of the Experimental Platform}

Test platform is built mainly by electronic nose system basic function module configuration based, generally include: sensor array, signal acquisition module, control module, the gas chamber temperature control module, controller, data storage module, communication interface, keyboard and display module and work independently, by training the built identification algorithm is given recognition results.

Working principle is through sampling system to be tested samples of the characteristics of the gas in the gas chamber, and then the signal acquisition module acquisition sensor array of gas sample response to pattern recognition unit gives the final judgment result.

The other main part of the experimental platform is the software system, which is the function of the system to complete the operation of the various control. According to the function of module, the system can be divided into main program, ADC driver, temperature and humidity sensor driver, LCD drive, measurement and control module, temperature control module, identification algorithm module and communication module.

\section{Description of the Experimental Process}

This test uses different quality state of the fourth tropical fruit agricultural product four sets of experiments (in this experiment were selected on behalf of the Hainan tropical fruit Australia mango, papaya, Sweetsop, banana as the experimental object), the volatile characteristics of gas component is very complex and gas sensor with cross sensitivity, so output signal of gas sensor array and can not directly for banana quality judgment. Therefore, it is necessary to select the effective data preprocessing technology and the intelligent pattern recognition to deal with the related data, including data processing, feature extraction, dimension reduction processing and recognition algorithm four parts.

Normalized sensor can make the output of each sensor in $[0,1]$, so that in response to each element of the vector is in the same order of magnitude can reduce calculation error recognition, and can make the input space of the neural network classifier to prepare the appropriate data.

The experiments for different quality state of Australia mango, papaya, Sweetsop, banana samples. The system 2 minutes of data acquisition, at $1 \mathrm{~Hz}$ sampling frequency of each sensor output 120 data, so each sample won the $120 \times 6$ data. The sample characteristic matrix, which is composed of the maximum value of each sensor's response, is a six dimensional data, and the computation quantity is still large when the pattern recognition is carried out. Therefore, the methods of PCA and LDA two were used to reduce the dimension of the feature data. Omit the dimensionality reduction step of the algorithm for BP algorithm; in reducing the dimension by PCA algorithm of data processing steps that algorithm for PCA+BP; down dimension using the LDA data processing step is called algorithm LDA+BP. And compared the results of the three different algorithms.

The samples of the sample quality identification test were 1 samples of normal samples, crushed samples, slightly corrupted or cracked samples The samples is shown Fig 1. Repeat test 3 times per sample. In contrast, 3 contrast experiments were done in clean air.

First sensor normalization processing, and then extract the maximum feature, and then compare the LDA and PCA dimension reduction effect, and finally compare the BP, PCA+BP and LDA+BP algorithm to determine the results.

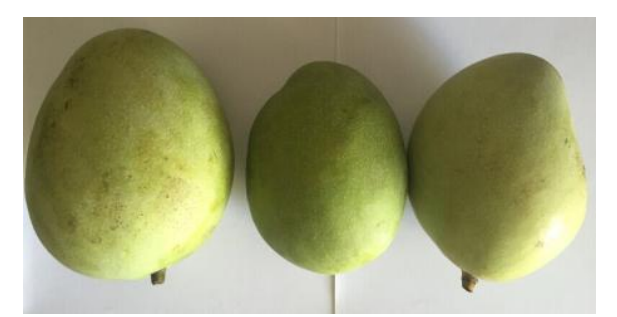

(1)Australia mango

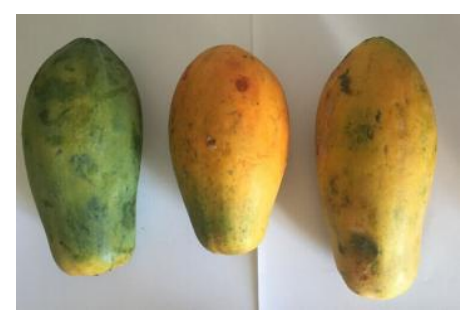

(2)Papaya 


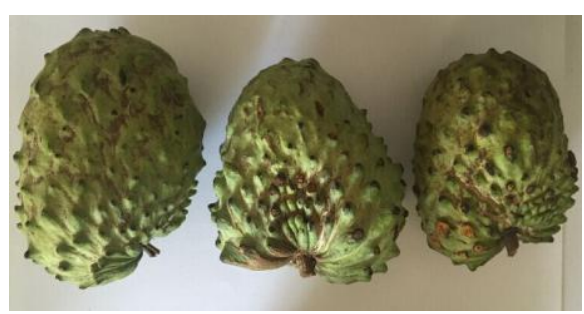

(3)Sweetsop

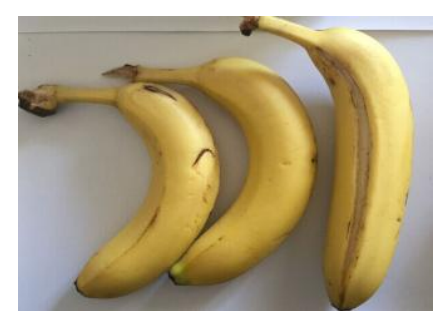

(4)Banana

Figure 1. Experiment samples

\section{Experimental Comprehensive Data Analysis}

From the result of the principal component analysis of the experimental data, two samples of each group to the quality of the gas principal component contribution rate were in more than $50 \%$, accumulative contribution rate reached $89.62 \%$ can think basically represent the basic information data. From the specific test data, it can be concluded that among all have obvious boundary, which air samples with each group of three samples of gas can clearly separated, and between the three types of samples, in addition to normal samples and the other two poor quality between the object class between distance outside, and the remaining two poor quality object class distance is nearly. This should be caused by the crushing of the sample object, the cracking of the sample object and the release of a number of similar characteristics of the gas caused by the.

After the normalization and feature extraction of the sensor response data, using LDA analysis method to reduce the dimensionality of the data processing and the processing of LDA. The changes of LD1 and were $86.34 \%$ and $10.13 \%$ respectively. The total contribution rate of LD2 and was $89.62 \%$, which could be used as the input signal of the BP neural network.

In the classification and discrimination experiments, the samples were divided into two groups: training samples and test samples. Each type of Australia mango, papaya, Sweetsop, banana were taken nine samples as training samples, in air in the three test samples as training samples, each sample is $9 * 3$ ten $2=29$ group and the rest of the group of samples as test samples. Using the original data, the data after PCA dimension reduction and the LDA dimension reduction data are used as the input of neural network, and the results of these three methods are compared. The number of hidden layer neurons is determined by the formula $\mathrm{l}=\sqrt{m+n}+\mathrm{a}, \mathrm{l}=\mathrm{is}[1,10]$, here take $\mathrm{a}=6$.

Eventually by the test samples tested results show that the algorithm uses a LDA+BP system identification accuracy rate of up to $100 \%$, can be effective in the three samples of each fruit sample state to distinguish, in order to achieve the purpose of quality detection.

Australia mango, papaya, Sweetsop, banana corruption process monitoring experiment samples for each group of three shapes are slightly different, the quality level of corruption is not the same, each sample test three times, a total of testing the 5-9 days, ranging from. The change process of the sample is shown Fig. 2.

Australia mango

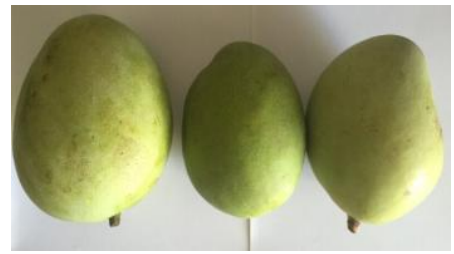

First days

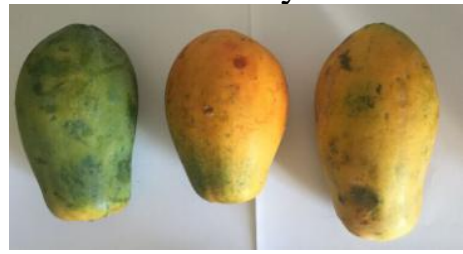

First days

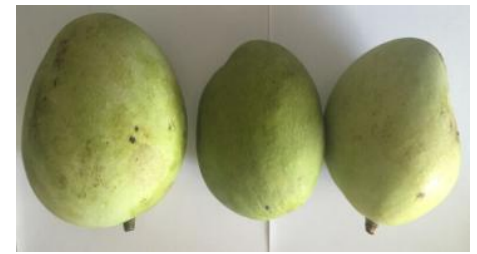

Fifth days

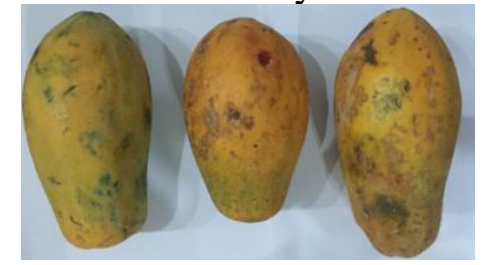

Third days

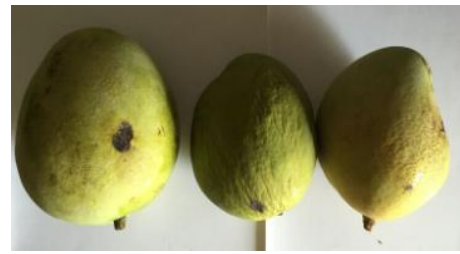

Ninth days

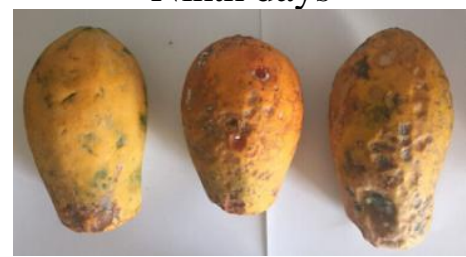

Fifth days 


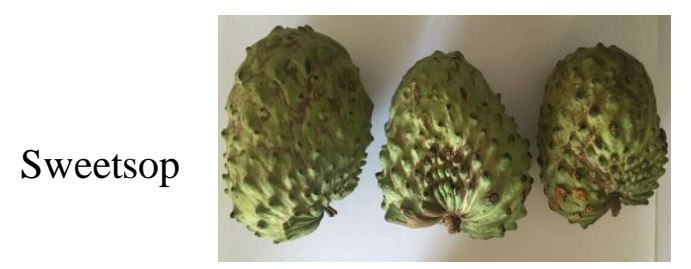

First days

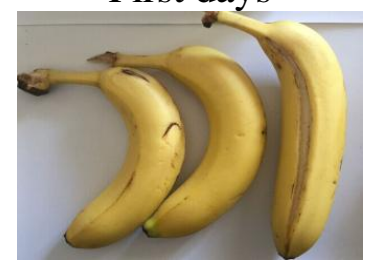

First days

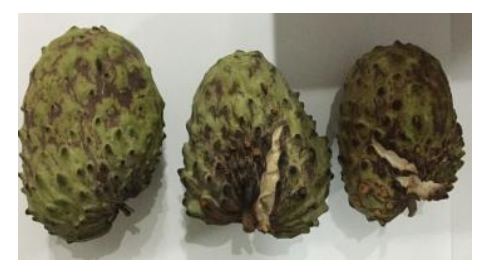

Third days

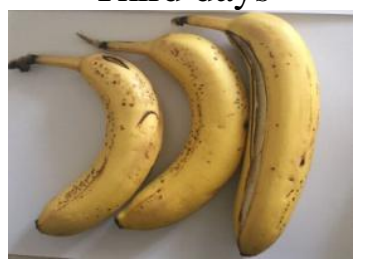

Fifth days

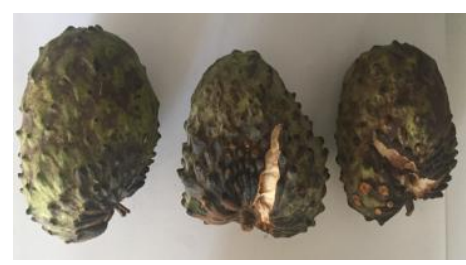

Fifth days

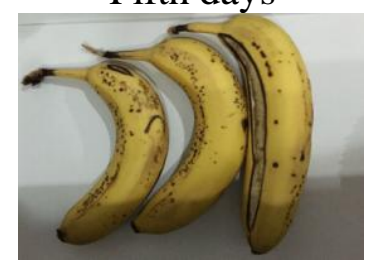

Seventh days

Figure 2. Experiment result image

From the experimental data of the general trend of view, in the process of corruption, with the process of corruption, the first principal component of the value of the data is increasing, and the value of the second principal component is a process of repeated shocks. This may be due to the complexity of the process of the corruption of the sample; the other may be due to the existence of a certain system of measuring errors.

Eventually by the test samples tested results show that the algorithm uses a LDA+BP system discriminant accuracy rate of up to $100 \%$, basic can effectively distinguish four kinds of tropical fruit samples at different stages of corruption, corruption of four tropical fruits samples could be predicted by. Using the BP neural network and PCA+BP method, the recognition accuracy was $76.23 \%$ and $86.35 \%$, respectively; the specific results are shown in the following Table 1 .

Table 1. Detection results of the three algorithms

\begin{tabular}{|l|c|c|c|}
\hline Algorithm & Error times & $\begin{array}{c}\text { Correct } \\
\text { number of } \\
\text { times }\end{array}$ & Accuracy rate \\
\hline BP neural network & 7 & 20 & $76.23 \%$ \\
\hline PCA+BP neural network & 4 & 23 & $86.35 \%$ \\
\hline LDA+BP neural network & 0 & 27 & $100 \%$ \\
\hline
\end{tabular}

The system can successfully identify the state of the different samples. Experimental results show that in the direct application of the BP neural network algorithm, our system identification correct rate is $76.23 \%$. And the PCA+BP neural network and LDA+BP neural network recognition algorithm, the system recognition correct rate $86.35 \%$ and $100.00 \%$, respectively.

In addition, the system has a certain effect on the prediction of the corruption of the four kinds of tropical fruits. After testing, the system uses LDA+BP algorithm to determine the accuracy rate of $96.22 \%$, the basic can effectively distinguish between different types of fruits of different stages of corruption, and you can predict the corruption of tropical fruits. At the same time, the BP neural network and PCA+BP method were used to predict the corruption of the same four kinds of tropical fruits, and the prediction accuracy was $75.69 \%$ and $87.52 \%$ respectively.

\section{Summary}

Due to the different categories of agricultural products and the release of odor gas is very complex, the system can only on the quality state of tropical agricultural products in tropical fruit is a basic classification, can not distinguish all the quality condition of the currently available to achieve a low cost simple categories are fewer test technology products. Based on the key technology of the electronic nose, and through optimization design makes the trend in the development of portable, 
seeking a specialized for tropical agricultural products quality inspection of portable electronic nose detection system, and ultimately the formation of products, applied to processes of warehousing and logistics is a good economic benefit and market application demand prospects.

\section{Acknowledgements}

The work was supported by the 2014 innovation and entrepreneurship training college national college projects(No. 201411100066, No. 201411100069); the Agricultural Science and Technology Innovation Project of Sanya (No. 2015KJ15, No. 2015KJ16, No. 2016NK17, No. 2016NK 18); the research project for Young Teachers of Qiongzhou university(No. QYQN201434).

\section{References}

[1] Yufei Wang, Meier Tan, Guixiang Xu, Experimental Study on the Detection of Tropical Fruit quality Based on Electronic Nose Technology, Software, Vol.37, No.3, pp. 40-43, 2016.

[2] Kun Zhang, Shengpei Dai, Based on the Electronic Nose Technology of Tropical Agricultural Product Quality Distinguish Detection Technology Research, Software, Vol.33, No.8, pp. 90-93, 2012.

[3] Kun Zhang, Shengpei Dai, Based on the Tropical Agricultural Product Quality Distinguish Experiment of Electronic, Software, Vol.34, No.2, pp. 88-89, 2013.

[4] Kun Zhang, Haifeng Wang, Shengpei Dai, Research on Tropical Agricultural Products Quality Recognition and Detection System based on Electronic Nose Technology, Advanced Materials Research. Vol. 659, pp. 75-78, 2013.

[5] JIN Jing, LIAO Gui-ping,LI Jin-wei,TONG Zhao, Survey on nondestructive detection to quality testing of agriculture products, Agriculture Network Information. , No.2, pp. 90-93, 2008.

[6] Jianfeng Zhu, Chengguo Yin, Zhibin Xiong, Design and Implementation of Quality Monitoring Information System of Agricultural Products, Journal of Qiongzhou University, Vol.18, No.5, pp. 17-21, 2011.

[7] Mansheng Long, Dongjian He, Jifeng Ning, An integrated apple grading system based on genetic neural network, Journal of Northwest Sci-Tech University of Agriculture and Forestry, Vol.29, No.6, pp. 108-111, 2001.

[8] Hongmei Zhang, Jun Wang, Optimization of sensor array of electronic nose and its application to detection of storage age of wheat grain, Transactions of the Chinese Society of Agricultural Engineering, Vol.22, No.12, pp. 164-167, 2006.

[9] Brezmes, J., E. Llobet, X.Vilanova, et al. Correlation between electronic nose signals and fruit quality indicators on shelf-life measurements with pink lady apples. 2001, Elsevier, pp. 41-50.

[10] Jonsson, A., F.Winquist, J.Sehnurer, et al, Electronic nose for microbial quality classification of grains, 1997, Elsevier, pp.187-193. 